\title{
Caracterización de la turba subtropical del departamento Islas del Ibicuy (Entre Ríos, Argentina)
}

\author{
Barbaro, L. A.; M. A. Karlanian; S. Imhoff y D. E. Morisigue
}

\begin{abstract}
RESUMEN
En la región del bajo Delta del Paraná (Argentina) se presentan condiciones anaeróbicas y en consecuencia, una acumulación de restos vegetales poco descompuestos que conforman la turba subtropical. Ésta es usada en el área metropolitana bonaerense como componente de sustratos para plantas, pero generalmente sus propiedades físicas y químicas son variables. Para hallar los factores que causan esta variación, el objetivo de este estudio fue caracterizar la turba subtropical del departamento Islas del Ibicuy. Se analizaron física y químicamente 27 muestras adquiridas en su lugar de origen y 51 muestras comerciales obtenidas en tres empresas. En la turba obtenida en su lugar de origen se observó desde la superficie a la base un aumento de la densidad, capacidad de retención de agua y CE, y una disminución de la materia orgánica, porosidad de aireación y pH. En las muestras comerciales se observaron mayores coeficientes de variación en los macro nutrientes. La turba subtropical extraída manualmente fue menos densa y con mayor contenido de materia orgánica que la extraída mecánicamente. La turba es variable a lo largo de su perfil, y su forma de extracción influye en sus propiedades.
\end{abstract}

Palabras claves: sustrato, delta del Paraná, turba subtropical.

Barbaro, L. A.; M. A. Karlanian; S. Imhoff and D. E. Morisigue, 2011. Characterization of the subtropical peat of the Islas del Ibicuy Department (Entre Ríos, Argentina). Agriscientia XXVIII (2): 137-145

\section{SUMMARY}

In the low Paraná Delta region (Argentina), anaerobic conditions promote the accumulation of vegetable wastes with almost no decomposition which form the subtropical peat. This is used in the Buenos Aires metropolitan area as a substrate component for plant production, but generally its physical and chemical properties are variable. The objective of this study was to characterize the subtropical peat from Islas Ibicuy Department in order to find the causes for this variation. Twenty-seven samples obtained in its original or natural place and fifty-one commercial samples obtained from three firms were physically 
and chemically analyzed. In the peat obtained from its place of origin an increasing density, water holding capacity and EC, and a decrease in organic matter, aeration porosity and $\mathrm{pH}$, was observed, from the surface to the base. In the commercial samples, greater variation coefficients in the macronutrient content were observed. Subtropical peat extracted manually was less dense and had more organic matter content than the one extracted mechanically. The peat is variable along the profile and the extraction system influences their properties.

Key words: Substrate, Paraná Delta, subtropical peat.

L. A. Barbaro, M. A. Karlanian y D. Morisigue. Instituto de Floricultura - INTA, De los Reseros y N. Repetto Hurlingham (1686), Pcia. Bs As, Argentina. S. Imhoff. Facultad de Ciencias Agrarias, UNL, Kreder 2805, Esperanza (S3080HOF), Pcia. Santa Fe, Argentina. Correspondencia a: Ibarbaro@cnia.inta.gov.ar

\section{INTRODUCCIÒN}

El delta del río Paraná, Argentina, se divide en tres regiones: el Delta Superior (desde Diamante, provincia de Entre Ríos, hasta Villa Constitución, provincia de Santa Fe), el Delta Medio (desde Villa Constitución hasta Ibicuy, provincia de Entre Ríos) y el Delta Inferior o bajo Delta (desde Ibicuy hasta la desembocadura en el río de la Plata). El bajo Delta es un humedal dulceacuícola que presenta una gran heterogeneidad ambiental, producto de su geomorfología y régimen hidrológico (Malvárez, 1999; Kandus et al., 2006). Kandus et al. (2006) identificaron en esta zona cinco unidades de paisaje, y la unidad dos, llamada bosques y praderas de los alrededores del río Paranacito, se caracteriza por suaves ondulaciones del terreno que corresponden a una sucesión de cordones arenosos separados por depresiones. Este paisaje se originó durante el retroceso del mar en un proceso de transgresión marina en el Holoceno y es retrabajado por la acción de las crecientes de los ríos Paraná y Uruguay (Kandus et al., 2006).

Hacia las zonas medias y bajas del gradiente topográfico se produce un reemplazo de las especies vegetales de acuerdo al aumento de las condiciones de anegamiento. La parte más deprimida del gradiente se encuentra inundada en forma permanente o por largos períodos de tiempo, estableciéndose un mosaico de especies del orden Poales dominado por Schoenoplectus californicus, que alternan con vegetación acuática flotante (Hydrochleis nymphoides, Myriophyllum aquaticum, Salvinia spp., Limnobium laevigatum) - arraigada (L. peruviana, Pontederia cordata, Sagittaria montevidensis y Ludwigia peploides) (Kandus et al., 2006).
Estas zonas inundables establecen condiciones anaeróbicas que generan acumulación de materia orgánica formada por restos vegetales poco descompuestos de las especies antes mencionadas (Malvárez, 1999). Estos restos se van depositando sobre ellos mismos, dependiendo la tasa de acumulación de materia orgánica de la productividad de la vegetación y de la tasa de descomposición, conformando la turba subtropical. La turba subtropical es también llamada resaca por los productores hortícolas y florícolas, pero actualmente se aconseja no llamarla de esta manera, debido a que la resaca es el limo o residuos que el mar o los ríos dejan en la orilla después de la crecida, por lo tanto, no es igual a la turba subtropical.

Turba es un nombre genérico que se aplica a diferentes materiales que proceden de la descomposición de los vegetales; la especie vegetal, su grado de descomposición, la variación entre climas locales y el agua determinan a su vez la calidad de la turba y su valor como componente de sustratos. La turba sphagnum, originada en ambientes fríos, es el material tradicional más utilizado como sustrato en la producción de plantas ornamentales en maceta (Mastalerz, 1977; Abad et al., 2001). Las características principales de la turba subtropical son similares a las de la turba sphagnum, y si bien en algunos casos presentan menor porosidad y mayor densidad que ésta, según los valores de referencia es un material potencialmente adecuado para su reemplazo (Di Benedetto et al., 2000; Gallardo, 2005).

La turba subtropical se usa en el Área Metropolitana de Buenos Aires (AMBA) como componente de sustratos para plantas en macetas, 
y proviene de la zona del Delta del río Paraná. Una desventaja de este material es su heterogeneidad. En análisis realizados en muestras de turba subtropical de varios productores y proveedores florícolas se encontraron diferentes valores para algunas variables, por ejemplo, $\mathrm{pH}$ entre 4,20 y 6,10 , conductividad eléctrica (CE) entre 0,14 dS $\mathrm{m}^{-1}$ y $1,23 \mathrm{dS}^{-1}$, y gran variabilidad en la concentración de cada nutriente (Barbaro et al., 2008).

Cuando el material utilizado para formular un sustrato es variable, se pueden alterar las características del producto final (Abad et al., 2004). Por ello, Mastalerz (1977) recomienda la estandarización del sustrato y de sus componentes, a efecto de asegurar que cada lote tenga las mismas propiedades físicas, químicas y biológicas. De lo contrario se podrían originar problemas con el riego, la fertilización y otras prácticas culturales.

Si se conociera en más detalle cuáles son los factores que contribuyen a la heterogeneidad de la turba subtropical sería posible prevenir o controlar esta desventaja. La turba subtropical utilizada en el AMBA proviene principalmente del departamento Islas del Ibicuy, al sudeste de la provincia de Entre Ríos, en el delta del río Paraná. Esta zona coincide con la unidad dos, descrita anteriormente.

El objetivo de este trabajo fue hallar los factores que influyen en la heterogeneidad de la turba subtropical, realizando la caracterización física y química de dos tipos de muestras: a) obtenidas in situ en el departamento Islas del Ibicuy, y b) provistas por diferentes empresas de sustratos que compran material en dicho departamento.

\section{MATERIALES Y MÉTODOS}

\section{Muestras obtenidas en el departamento Islas del Ibicuy}

Muestras de turba subtropical extraídas manualmente

Se tomaron muestras de turba formada en un sector del arroyo Las Animas ( $33^{\circ} 34^{\prime} 42^{\prime \prime}$ S; $58^{\circ}$ $37^{\prime} 10^{\prime \prime}$ O), en una formación de hasta 1,8 m de profundidad que cubría la superficie del arroyo. El muestreo se realizó manualmente mediante serruchos especiales, obteniendo bloques de turba de 1,80 $\mathrm{m}$ de profundidad $\times 0,70 \mathrm{~m}$ de ancho y $0,40 \mathrm{~m}$ de largo. Del total de bloques extraídos el día del muestreo se tomaron tres al azar y a cada uno se lo dividió, desde la superficie a la base, en seis partes transversales de $30 \mathrm{~cm}$ cada una, donde cada parte fue una muestra. En total se tomaron 18 muestras, 6 por cada bloque de turba subtropical.

Muestras de turba subtropical extraídas en forma mecánica

Se tomaron muestras de turba formada en

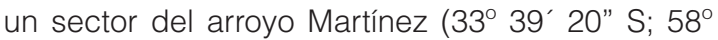
$41^{\prime} 42$ " O), en una formación de hasta $1 \mathrm{~m}$ de profundidad. Para extraerla, se dragó el agua de una sección del arroyo y la turba formada en su superficie se depositó sobre el piso formado por sedimentos. Se tomaron las muestras antes de que la turba sea extraída con palas mecánicas, eligiendo tres lugares al azar. En cada lugar se tomaron tres muestras con palas manuales desde la superficie a la base de la turba, llamándolas parte superior $(30 \mathrm{~cm})$, media $(60 \mathrm{~cm})$ e inferior (90 $\mathrm{cm})$. En total se tomaron nueve muestras, tres por cada lugar.

\section{Muestras de sedimentos}

Luego de extraer la turba mecánicamente se continuaron extrayendo los sedimentos, uno superior de coloración marrón y otro inferior de coloración gris. En ese momento, se tomaron muestras de cada uno en los tres lugares en que se extrajeron las muestras de turba.

\section{Muestras de turba subtropical comercial}

Durante el año 2009 se tomaron muestras en tres empresas proveedoras de turba subtropical en la zona del AMBA, las cuales compran turba en el departamento Islas del Ibicuy. Se realizaron visitas mensuales a las empresas para tomar las muestras, aunque hubo meses en que se recolectaba una muestra de solo una pila y otros de varias, o de ninguna porque no había turba en ese momento. La turba subtropical se encontraba depositada en pilas; por tal motivo, para tomar una muestra se recolectó $5 \mathrm{~L}$ de turba en 10 puntos diferentes. Una vez totalizados los $50 \mathrm{~L}$ se mezcló y se dividió en cuatro partes de $12,5 \mathrm{~L}$ cada uno y de cada parte se tomaron $3 \mathrm{~L}$. De esta forma se conformó una muestra compuesta de $12 \mathrm{~L}$ y se llevó al laboratorio para realizar los correspondientes análisis físicos y químicos.

Al finalizar el muestreo, en total se tomaron 51 muestras, 8 en la empresa 1, 19 en la empresa 2 y 24 en la empresa 3.

\section{Análisis químicos y físicos}

Las muestras se analizaron en el laboratorio de sustratos y aguas del Instituto de Floricultura del INTA (Instituto Nacional de Tecnología 
Agropecuaria). Las variables medidas y las metodologías empleadas fueron:

$\mathrm{pH}$ y conductividad eléctrica (CE) $1+5 \mathrm{v} / \mathrm{v}$ : En un frasco de $250 \mathrm{~mL}$ se colocaron $150 \mathrm{~mL}$ de agua destilada y se llevó a $180 \mathrm{~mL}$ con el agregado de sustrato. Se agitó durante 10 minutos y se dejó reposar 15 minutos, luego se realizaron las mediciones en la solución con un peachímetro (Horiba M-12) y un conductímetro (Thermo Orion modelo 145).

Macro y micro elementos solubles: Sobre el extracto de la solución filtrada $(1+5 \mathrm{v} / \mathrm{v})$, se analizó el contenido de calcio, magnesio, potasio, sodio, zinc, manganeso, cobre y hierro con un espectrofotómetro de absorción atómica (Marca Varian modelo 220 A), y los nitratos con un electrodo de ión selectivo (Marca Orion Modelo 920 A). Los micronutrientes no fueron analizados en las turbas subtropicales comerciales.

Materia orgánica (MO): Fue determinado mediante el método de la mufla (Ansorena, 1994).

Densidad aparente: Se determinó con el método descrito por Hoffmann (1970).

Espacio poroso total (EPT), capacidad de retención de agua (CRA) y poros con aire (PA): Estas variables se determinaron con el método propuesto por De Boodt et al. (1974), mediante los lechos de arena.

Granulometría: Mediante una tamizadora con tamices de $5,56 \mathrm{~mm}, 4,75 \mathrm{~mm}, 3,35 \mathrm{~mm}, 2 \mathrm{~mm}$, $1,4 \mathrm{~mm}, 1 \mathrm{~mm}$ y $0,5 \mathrm{~mm}$ (Ansorena, 1994). Este análisis se realizó solo a las muestras de turba subtropical comercial.

\section{Análisis estadístico}

Los datos fueron analizados con un software estadístico Infostat Profesional versión 2009. Se utilizó la prueba de Tukey $(p \leq 0,05)$ para comparación de medias, se hicieron análisis de correlación de Pearson, se calcularon coeficientes de variación y se hizo un análisis de componentes principales utilizando todas las variables medidas a las muestras de turba subtropical comercial.

\section{RESULTADOS Y DISCUSION}

\section{Muestreo de turba subtropical en forma mecá- nica y manual}

Las determinaciones físicas evaluadas en cada parte de la formación de turba subtropical desde la superficie a la base variaron debido a que los sedimentos traídos por el agua se depositaron y se acumularon en la base de la turba, especialmente la extraída en forma mecánica. La materia orgánica disminuyó hacia la parte inferior de la turba y en consecuencia, la densidad aparente aumentó, por lo que se encontraron diferencias significativas entre la última porción del bloque de turba con respecto a los restantes. Esta última porción fue la de menor porcentaje de materia orgánica y mayor densidad (Figuras 1 y 2 ).

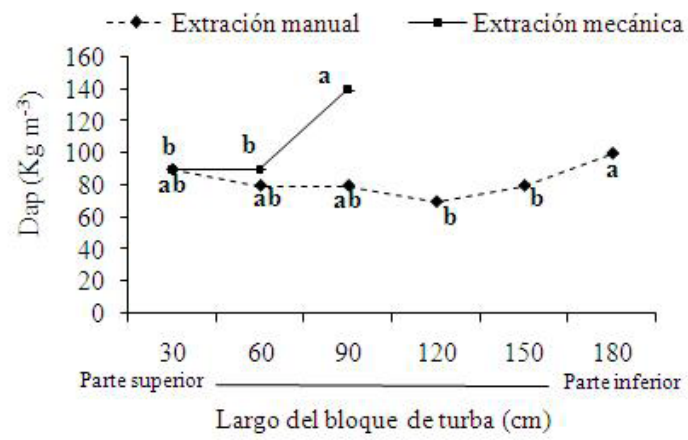

Figura 1. Densidad aparente (Dap) a lo largo del bloque de turba subtropical extraída en forma manual y mecánica en el departamento Islas del Ibicuy. Barras verticales en cada punto indican intervalo de confianza de 95\%. Letras distintas en una línea indican diferencias significativas $(p \leq 0,05)$

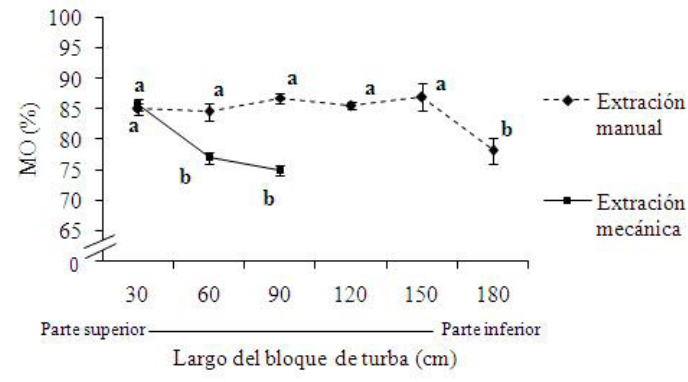

Figura 2. Materia orgánica $(\mathrm{MO})$ a lo largo del bloque de turba subtropical extraída en forma manual y mecánica en el departamento Islas del Ibicuy. Barras verticales en cada punto indican intervalo de confianza de 95\%. Letras distintas en una línea indican diferencias significativas $(p \leq 0,05)$.

También se observó disminución de la porosidad de aireación e incremento de la capacidad de retención de agua hacia la base de la turba en ambos tipos de extracción (Figura 3). Los sedimentos traídos por el agua contienen partículas finas que se mueven dentro de los espacios libres disminuyendo los poros con aire. Por lo tanto, al haber poros más chicos se incrementa la capacidad de retención de agua (Ansorena, 1994; Fernández y Corá, 2004). Además, la turba de la 


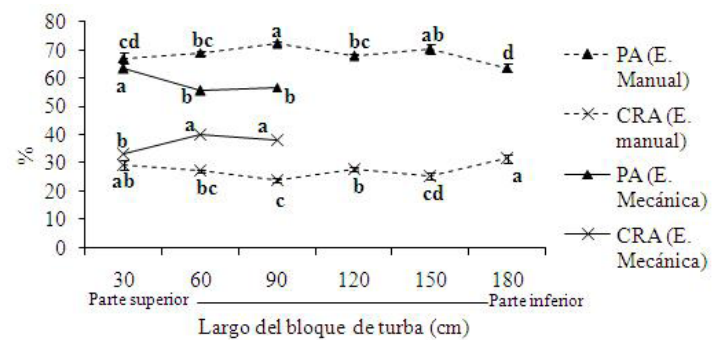

Figura 3. Porosidad de aireación (PA) y capacidad de retención de agua (CRA) a lo largo del bloque de turba subtropical extraída en forma manual (E. manual) y mecánica (E. mecánica) en el departamento Islas del Ibicuy. Barras verticales en cada punto indican intervalo de confianza de 95\%. Letras distintas en una línea indican diferencias significativas $(p \leq 0,05)$.

parte inferior suele estar más descompuesta y generalmente tiene un tamaño menor de partículas (Burés, 1997). Si bien la relación de poros con aire y capacidad de retención de agua varió, el espacio poroso total no se diferenció a lo largo de la turba manteniéndose en $96 \%$ en la extraída manualmente y en $95 \%$ en la extraída mecánicamente.

Con respecto a las propiedades químicas, hubo disminución del $\mathrm{pH}$ y aumento de la CE principalmente en la base de los bloques de turba extraída de ambas formas (Tabla 1). Los valores de $\mathrm{CE}$ encontrados no fueron altos, ya que materiales con valores menores a $1 \mathrm{dS} \mathrm{m}-1(\mathrm{v} / \mathrm{v})$ son adecuados para su uso como sustrato (Abad et al., 1993).

En los suelos hidromorfos, en este caso un suelo orgánico, se suelen evidenciar condiciones reductoras como consecuencia de la saturación hídrica, que generan un prolongado déficit en oxigeno libre. En este caso, los microorganismos aerobios se vuelven latentes o mueren, mientras que los anaerobios se multiplican y descomponen la materia orgánica utilizando compuestos oxidados para su respiración. Como resultado, se producen cambios en el $\mathrm{pH}$, disminución del potencial redox y aumento de la CE (CIAT, 1981; Pereyra et al., 2004). Dichas condiciones también facilitan que ciertos nutrientes como el magnesio, manganeso y hierro estén disponibles en sus formas reducidas (Malvárez, 1999).

La presencia de sedimentos entre la turba también tuvo influencia en las propiedades químicas de la turba subtropical. El valor de $\mathrm{pH}$ de los sedimentos fue de 4 y de los elementos analizados hubo mayores niveles de calcio, sodio y magnesio entre los macronutrientes y manganeso entre los micronutrientes (Figura 4).

El incremento de la CE hacia la parte inferior de

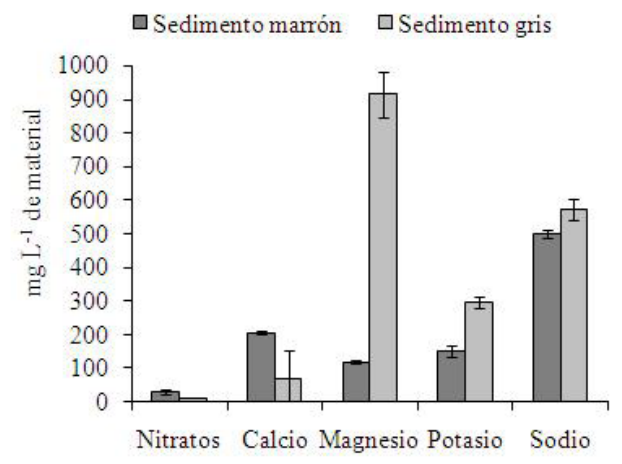

A

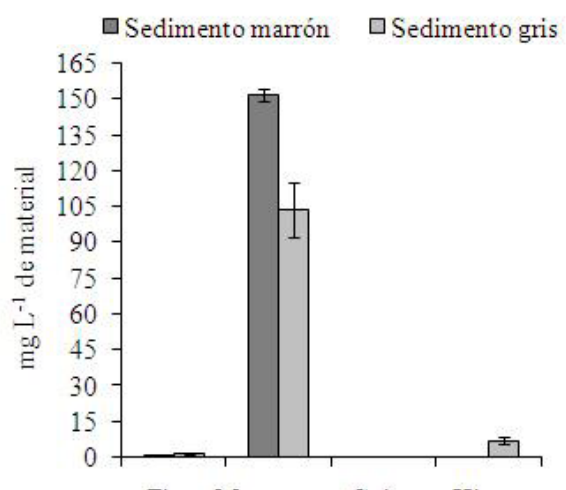

Zinc Manganeso Cobre Hierro

\section{B}

Figura 4. A) Nitratos, calcio, magnesio, potasio y sodio del sedimento marón y gris. B) Zinc, manganeso, cobre y hierro del sedimento marón y gris. Barras verticales en cada punto indican intervalo de confianza de $95 \%$.

Tabla 1. pH y conductividad eléctrica (CE) de las diferentes partes del bloque de turba subtropical extraída en forma manual y mecánica en el departamento Islas del Ibicuy.

\begin{tabular}{lccccccccc} 
& \multicolumn{4}{c}{ Partes del bloque de turba subtropical } \\
\cline { 2 - 10 } & 1 & 2 & 3 & 4 & 5 & 6 & Superior & Medio & Inferior \\
\hline $\mathrm{pH}$ & 5,5 & 5,3 & 5,3 & 5,3 & 5,1 & 4,2 & 4,0 & 3,8 & 3,6 \\
$\mathrm{CE}\left(\mathrm{dS} \mathrm{m}{ }^{-1}\right)$ & 0,26 & 0,12 & 0,12 & 0,14 & 0,15 & 0,90 & 0,3 & 0,3 & 1,1 \\
\hline
\end{tabular}


la turba con ambas formas de extracción, tuvo un coeficiente de correlación de 0,86 con el sodio, 0,96 con el calcio, 0,99 con el magnesio y 0,96 con el manganeso. Es decir, que el incremento del volumen de sedimento hacia la base de la turba coincide con el incremento de la conductividad eléctrica de ésta, debido a los elementos de mayor concentración que se encuentran en los sedimentos (Figuras 5 y 6 ).

Las islas del Delta del Paraná se formaron principalmente por la gran cantidad de sedimentos que acarrea el agua del río Paraná; éste transporta hacia el río de la Plata 160 millones de toneladas anuales de sedimentos, de los cuales 50 a $70 \%$ provienen principalmente de la alta cuenca del río Bermejo (Depetris et. al., 2003). La presencia de calcio, magnesio, sodio y manganeso que se destacan en los sedimentos evaluados, se debe a su composición mineralógica. Varios autores han examinado en los últimos 30 años las características químicas del río Paraná, y concluyeron que en orden de abundancia se encuentran entre los aniones los bicarbonatos, cloro y sulfatos, y entre los cationes el sodio, calcio y magnesio (Depetris et al., 2003).

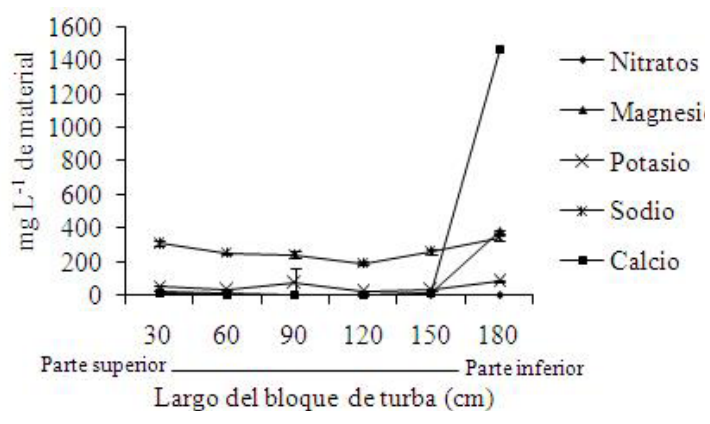

A

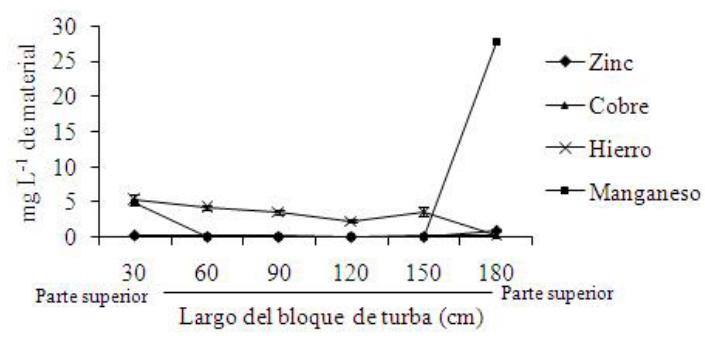

\section{B}

Figura 5. A) Nitratos, calcio, magnesio, potasio y sodio de las diferentes partes del bloque de turba subtropical extraída en forma manual en el departamento Islas del Ibicuy. B) Zinc, manganeso, cobre y hierro de turba subtropical extraída en forma manual en el departamento Islas del Ibicuy. Barras verticales en cada punto indican intervalo de confianza de 95\%.

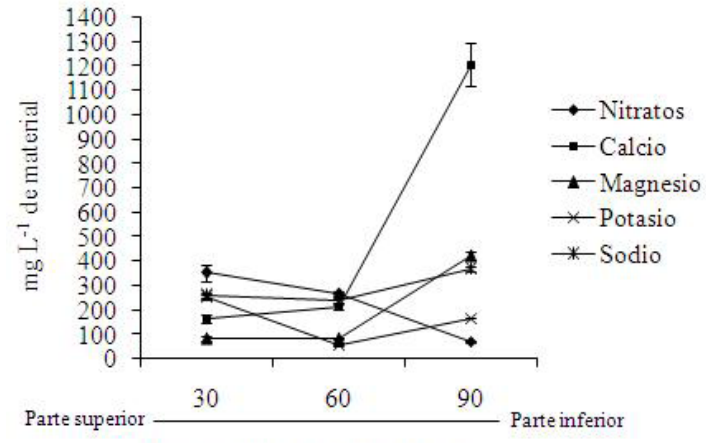

Largo del bloque de turba $(\mathrm{cm})$

A

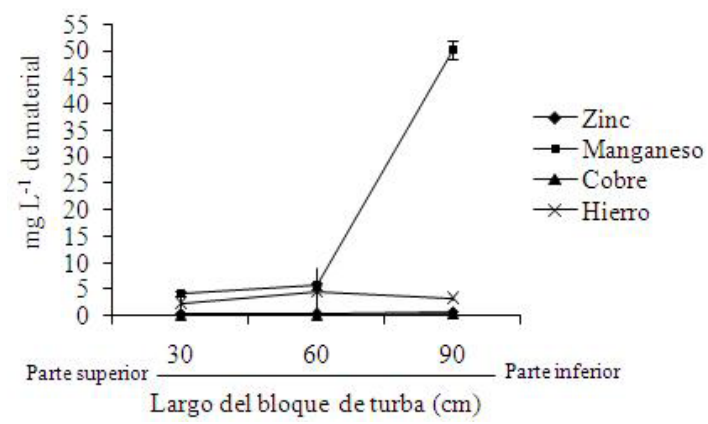

B

Figura 6. A) Nitratos, calcio, magnesio, potasio y sodio de las diferentes partes del bloque de turba subtropical extraída en forma mecánica en el departamento Islas del Ibicuy. B) Zinc, manganeso, cobre y hierro de turba subtropical extraída en forma manual en el departamento Islas del Ibicuy. Barras verticales en cada punto indican intervalo de confianza de $95 \%$.

\section{Muestreo de turba subtropical comerciali- zada por las empresas}

Al analizar las muestras de turba subtropical comercializada por tres empresas, se pudo corroborar la variabilidad que presenta este material (Tabla 2), en especial en el contenido de macro nutrientes.

En estas muestras se destacan los menores niveles de cada nutriente con respecto a los encontrados en las muestras de turba extraída en su lugar de origen. En las empresas, la turba estaba acopiada en pilas sin resguardo de las condiciones atmosféricas, y teniendo en cuenta que una forma de reducir la presencia sales solubles es mediante lixiviación (Abad et al., 2004), es posible que las precipitaciones hayan contribuido al lavado de la turba. La densidad aparente promedio fue similar a las encontradas por Lucas et al. (1965), revalidando que las turbas subtropicales formadas 
Tabla 2. Media, coeficiente de variación (CV), mínimo y máximo de muestras de turba subtropical comercial.

\begin{tabular}{lcccc}
\hline \multicolumn{1}{c}{ Variable } & Media & CV & Mínimo & Máximo \\
\hline $\mathrm{pH}$ & 4,9 & 6 & 4,4 & 5,8 \\
$\mathrm{CE}^{*}\left(\mathrm{dS} \mathrm{m}{ }^{-1}\right)$ & 0,6 & 39 & 0,1 & 1,0 \\
Nitratos $\left(\mathrm{mg} \mathrm{L}^{-1}\right)$ & 230 & 93 & 0 & 749 \\
Calcio $\left(\mathrm{mg} \mathrm{L}^{-1}\right)$ & 220 & 88 & 10 & 770 \\
Magnesio $\left(\mathrm{mg} \mathrm{L}^{-1}\right)$ & 167 & 77 & 8 & 471 \\
Potasio $\left(\mathrm{mg} \mathrm{L}^{-1}\right)$ & 318 & 54 & 102 & 906 \\
Sodio $\left(\mathrm{mg} \mathrm{L}^{-1}\right)$ & 404 & 49 & 131 & 915 \\
Dap. ${ }^{*}\left(\mathrm{Kg} \mathrm{m}^{-3}\right)$ & 200 & 42 & 100 & 300 \\
MO* $\left.^{*} \%\right)$ & 56 & 23 & 34 & 93 \\
PA* $\left.^{*} \%\right)$ & 43 & 40 & 10 & 72 \\
CRA $^{*}(\%)$ & 47 & 30 & 22 & 75 \\
EPT* $(\%)$ & 90 & 5 & 80 & 98 \\
\hline
\end{tabular}

* Conductividad eléctrica (CE), densidad aparente (Dap), poros con aire (PA), espacio poroso total (EPT), capacidad de retención de agua (CRA), materia orgánica $(\mathrm{MO}) \mathrm{N}=51$

por juncos son hasta 2,5 veces más densas que la turba formada por musgo sphagnum.

Al analizar las 51 muestras de turba subtropical con todas sus variables se obtuvieron cuatro componentes principales (CP), que explicaron el $74 \%$ de la variabilidad de los datos. Se obtuvieron dos gráficos biplot uno con las CP1 y CP2 (Figura 7), y otro con las CP3 y CP4 (Figura 8). La CP1, conformada por las variables densidad aparente, porcentaje de partículas $<1 \mathrm{~mm}$ y conductividad eléctrica con los coeficientes positivos más altos y la materia orgánica, espacio poroso total y porosidad de aire con los coeficientes negativos más altos, explicó el 39\% de la variabilidad; la CP2, conformada por las variables magnesio, calcio y nitratos con los coeficiente positivos más alto y la variable $\mathrm{pH}$ con el coeficiente negativo más alto, explicó el 17\% de la variabilidad; la CP3, conformada por las variables $\mathrm{pH}$ y porcentaje de partículas entre 3,35 y $1 \mathrm{~mm}$ con los coeficientes positivos más alto y las variables porcentaje de partículas $>3,35 \mathrm{~mm}$ y capacidad de retención de agua con los coeficientes negativos más altos, explicó el 9\%, igual que la CP4, conformada por las variables fósforo y sodio con los coeficientes positivos más altos y calcio con el coeficiente negativo más alto.

En este análisis se pudo destacar que la CP1 separó dos grupos de muestras de turba subtropical comercial; por un lado, las que contenían mayor porcentaje de materia orgánica, y por el otro, las que contenían mayor densidad aparente. Todas las muestras de la empresa 3, dos muestras de la empresa 2 y la mitad de las muestras de la empresa 1 tuvieron mayor porcentaje de materia orgánica. En cambio, todas las muestras de la empresa 2, excepto dos muestras, y la otra mitad de muestras de la empresa 1, fueron las de mayor densidad aparente. A su vez, la CP2 separó dos nuevos grupos, uno con muestras de mayor contenido de magnesio y otro con muestras con mayor valor de $\mathrm{pH}$.

Las muestras se agruparon según su forma de extracción, ya que la empresa 2 obtiene turba extraída mecánicamente, la empresa 3 adquiere turba extraída manualmente y la empresa 1 de

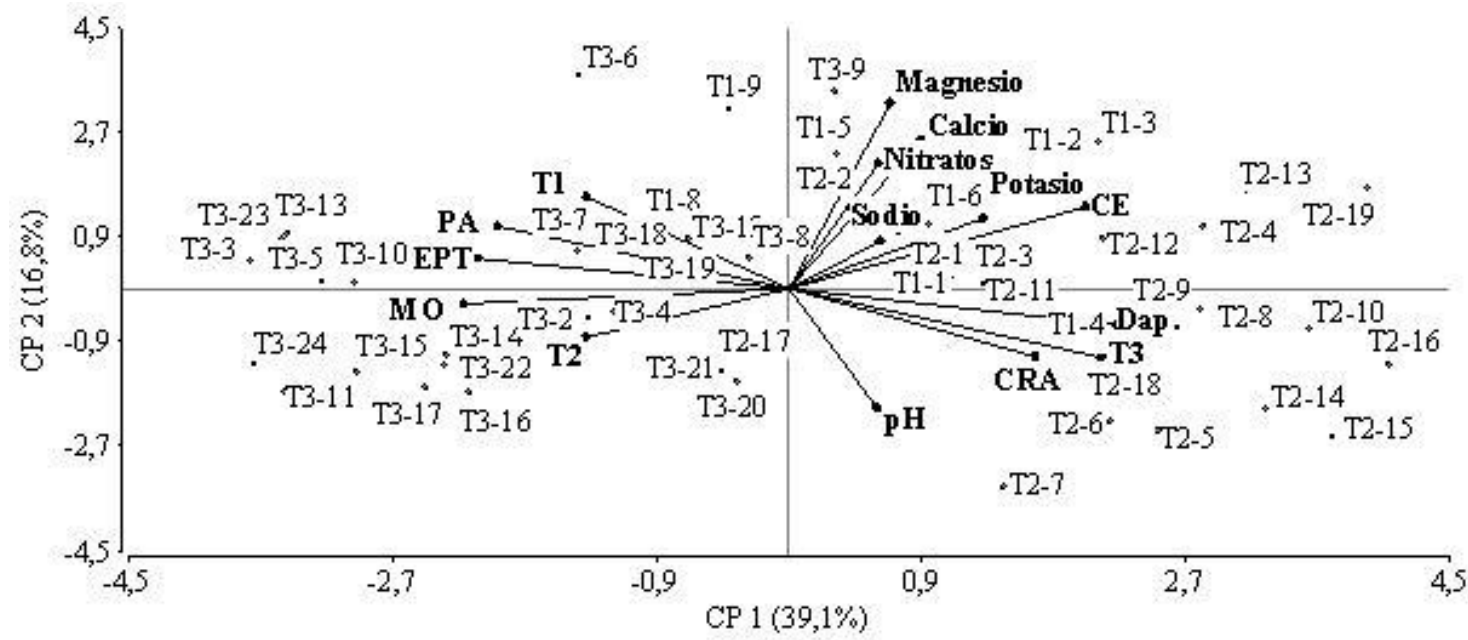

Figura 7. Biplot conformado por dos componentes (CP1 y CP2) generadas por la combinación lineal de las variables porcentaje de partículas > 3,35 mm (T1), porcentaje de partículas 3,35-1 mm (T2), porcentaje de partículas < $1 \mathrm{~mm}$ (T3), materia orgánica (MO), densidad aparente (Dap.), porosidad d aireación (PA), capacidad de retención de agua (CRA), espacio poroso total (EPT), pH, conductividad eléctrica (CE), calcio, magnesio, potasio, sodio y nitratos de las muestras de turba subtropical. *Turba subtropical de la empresa 1 (T1), turba subtropical de la empresa 2 (T2), turba subtropical de la empresa 3 (T3). 


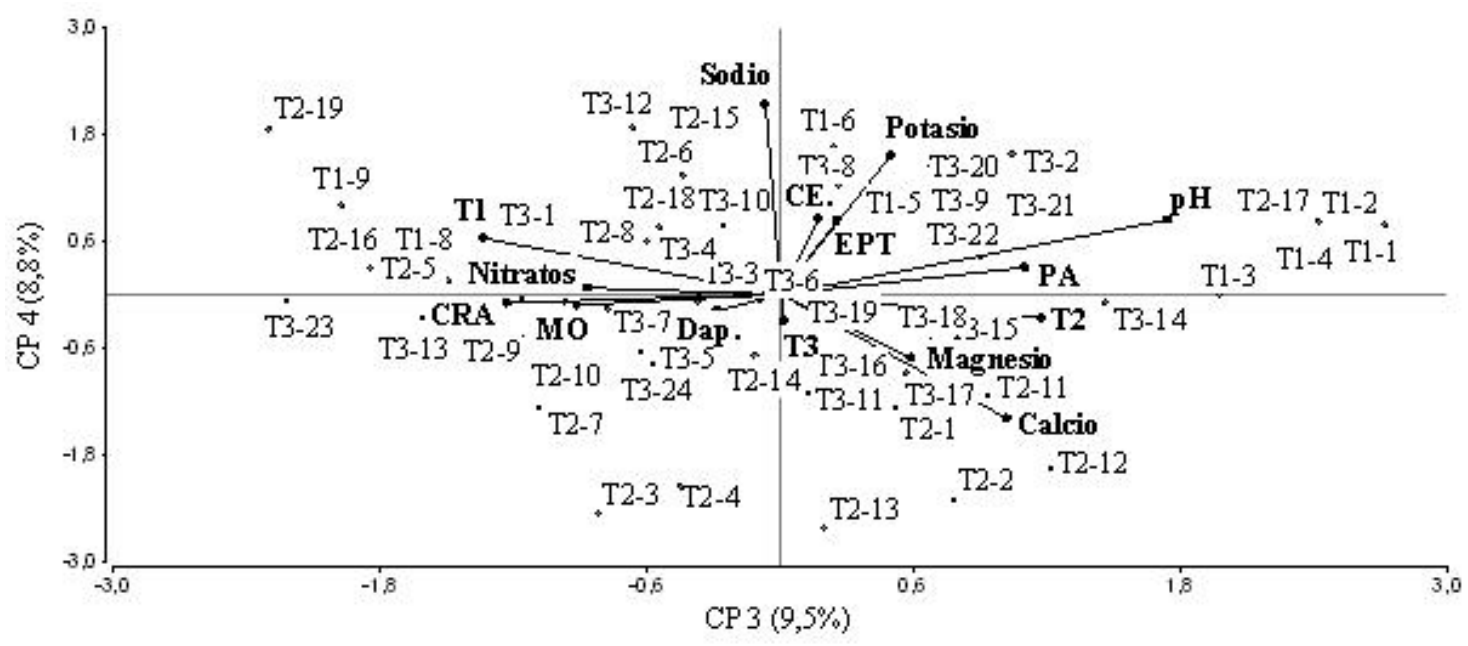

Figura 8. Biplot conformado por dos componentes (CP1 y CP2) generadas por la combinación lineal de las variables porcentaje de partículas > 3,35 mm (T1), porcentaje de partículas 3,35-1 mm (T2), porcentaje de partículas < $1 \mathrm{~mm}$ (T3), materia orgánica $(\mathrm{MO})$, densidad aparente (Dap.), porosidad d aireación (PA), capacidad de retención de agua (CRA), espacio poroso total (EPT), pH, conductividad eléctrica (CE), calcio, magnesio, potasio, sodio y nitratos de las muestras de turba subtropical. *Turba subtropical de la empresa 1 (T1), turba subtropical de la empresa 2 (T2), turba subtropical de la empresa 3 (T3).

ambas formas. Esto permite inferir que la turba subtropical extraída manualmente fue menos densa, con mayor contendido de materia orgánica y, según el 70\% de las muestras, tenía mayor $\mathrm{pH}$, aunque igualmente son valores ácidos. La turba extraída en forma mecánica fue más densa, con menor contenido de materia orgánica y un $63 \%$ de las muestras tuvieron mayores niveles de magnesio. Estos resultados coinciden con los obtenidos en las muestras tomadas en su lugar de origen con cada forma de extracción. Wilson (1985) también encontró variaciones en las características físicas de la turba de musgo según el tipo de extracción, principalmente en la porosidad. En este caso, el minado hidráulico produce rompimiento de la estructura de las partículas de la turba de musgo, por lo que aconseja cortar en bloques. Actualmente muchas compañías canadienses rastrillan la turba de musgo en los primeros centímetros y luego aspiran la turba suelta, pero se observó que reduce el tamaño de las partículas (Reed, 1999). Por lo tanto, la forma de extracción tiene influencia en las propiedades físicas y químicas de la turba.

\section{CONCLUSIÓN}

La turba subtropical es variable desde su origen. La parte superior de la formación de turba tiene propiedades distintas a la inferior, debido principalmente a la presencia de sedimentos y a las condiciones anaerobias del medio.
La forma de extracción de la turba subtropical influye en las propiedades que tendrá la turba una vez obtenida, pudiendo incrementar el contenido de sedimentos.

Antes de obtener la turba se debería tener en cuenta el origen y la forma de extracción. Una vez obtenida, es importante mezclar todo el lote para homogeneizar y realizar un análisis físico/químico.

\section{AGRADECIMIENTOS}

Al Municipio de Villa Paranacito y a la agencia del INTA por facilitar el estudio en la zona. A los Sres. Donag y Godoy por permitir tomar muestras de turba subtropical en sus propiedades y gentilmente responder todas las inquietudes. A las empresas Terrafértil S.A., Bertinat y Jardín Florido por la colaboración recibida. A Diego Mata por sus valiosas sugerencias.

\section{BIBLIOGRAFIA}

Abad B., M.; P. F. Martínez; M. D. Martínez y J. Martínez, 1993. Evaluación agronómica de los sustratos de cultivo. Actas de Horticultura 11: 141-154.

Abad B., M., P. Noguera and S. Burés, 2001. National inventory of organic wastes for use as growing media for ornamental potted plant production: case study in Spain. Bioresource Technol. 77: 197-200.

Abad B., M.; P. Noguera y C. Carrion, 2004. Los sustratos en los cultivos sin suelo. In: Urrestarazu G., M. (Ed), 
2004. Tratado de Cultivo sin Suelo. Ediciones Mundi Prensa. pp. 113-158.

Ansorena M., J., 1994. Sustratos Propiedades y Caracterización. Ed. Mundi-Prensa. Madrid. 172 pp.

Barbaro, L. A., M. A. Karlanian y D. E. Morisigue, 2008. Caracterización química de resacas de río utilizadas para la formulación de sustratos. In: Memoria del VI Encontro Nacional sobre Substratos para Plantas. 15-18 septiembre. Fortaleza, Brasil. (CD). pdf 18. pp. 188-190.

Burés, S., 1997. Sustratos. Ed. Agrotecnias. Madrid. 342 pp.

CIAT (Centro Internacional De Agricultura Tropical), 1981. Química de los suelos inundados. Serie 04SR-09.01. $36 \mathrm{pp}$.

Depetris P.J., J.L. Probst, A.I. Pasquini and D.M. Gaiero, 2003. The geochemical characteristics of the Paraná River suspended sediment load: an initial assessment. Hydrological Processes 17: 1267-1277.

Di Benedetto, A., J. Molinari, C. Boschi, R. Klasman y D. Benedicto, 2000. Adaptación de cuatro especies florales anuales a diferentes sustratos de crecimiento. Agro Sur28.http://mingaonline. uach.cl/scielo.php?script=sci_arttext\&pid=S0304$88022000000200009 \&$ lng $=$ es\&nrm=iso. Consultado el 7-11-2007.

Fernández, C. y J. E. Corá, 2004. Densidade e relacao ar/agua de substratos hortícolas. Sicentia Agricola 61. $N^{\circ} 4: 446-450$.

Gallardo, S. C., 2005. Bases Técnicas para Elección de los Sustratos: Problemas y Soluciones más comunes. Boletín de Floricultura. № 35 . Disponible en http://
www.inta.gov.ar/sanpedro/info/Bflori/035_bf.htm\#5. Consultado el 10-3-2010

Hoffmann, G., 1970. Verbindlinche Methoden zur Untersuchung von RKSund Gartnerischen Erden. Miteilubngen der VDLUFA, Heft. pp. 129-153.

Infostat, 2009. Versión profesional. Estadística y Biometría, Facultad de Ciencias Agropecuarias, Universidad Nacional de Córdoba.

Kandus, P., R. D. Quintana y R. F. Bó 2006. Patrones de Paisaje y Biodiversidad del Bajo Delta del Río Paraná. $1^{\circ}$ edición. Pablo Casamajor Ediciones. Buenos Aires. $38 \mathrm{pp}$.

Lucas, R. E., P. E. Rieke y R. S. Farmham, 1965. Peats for soil improvement and soil mixes. Farm Sci. Ser. Ext. Bull. 516. Lansing, MI: Michigan State University, Cooperative Extension Service. 11 pp.

Malvárez, A.l., 1999. Tópicos sobre Humedales Subtropicales y Templados de Sudamérica. Oficina regional de ciencia y tecnología de la UNESCO para América latina y el Caribe. Ed. UBA. 228 pp.

Mastalerz, J. W., 1977. The Greenhouse Environment. New York. John Wileyand Sons. 629 pp.

Pereyra, F. X., V. Baumann, V. Altinier, J. Ferrer y P. Tchilinguirian, 2004. Génesis de suelos y evolución del paisaje en el delta del Río Paraná. Revista de la Asociación Geológica Argentina 59, № 2: 229-242.

Reed, D. W., 1999. Guía del Productor, Agua, Sustratos y Nutrición en los Cultivos de Flores bajo Invernadero. Ed. Ball Publishing y Hortitecnia Itda. 305 pp.

Wilson G., C. S., 1985. Effects of additives to peat on the air and water capacity. Acta Horticulturae 172:207209. 